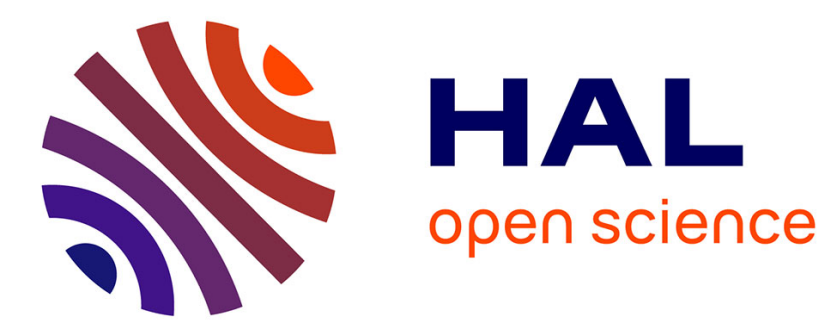

\title{
More insight in multiple bonding with valence bond theory
}

\author{
K. Hendrickx, B. Braida, P. Bultinck, P.C. Hiberty
}

\section{To cite this version:}

K. Hendrickx, B. Braida, P. Bultinck, P.C. Hiberty. More insight in multiple bonding with valence bond theory. Computational and Theoretical Chemistry, 2015, 1053, pp.180-188. 10.1016/j.comptc.2014.09.007 . hal-01627698

\section{HAL Id: hal-01627698 \\ https://hal.science/hal-01627698}

Submitted on 21 Nov 2017

HAL is a multi-disciplinary open access archive for the deposit and dissemination of scientific research documents, whether they are published or not. The documents may come from teaching and research institutions in France or abroad, or from public or private research centers.
L'archive ouverte pluridisciplinaire HAL, est destinée au dépôt et à la diffusion de documents scientifiques de niveau recherche, publiés ou non, émanant des établissements d'enseignement et de recherche français ou étrangers, des laboratoires publics ou privés. 


\title{
More insight in multiple bonding with valence bond theory
}

\author{
K. Hendrickx ${ }^{\mathrm{a}, \mathrm{b}, \mathrm{c}}$, B. Braida ${ }^{\mathrm{a}, \mathrm{b}, *}$, P. Bultinck ${ }^{\mathrm{c}}$, P.C. Hiberty ${ }^{\mathrm{d}, *}$ \\ a Sorbonne Universités, UPMC Univ Paris 06, UMR 7616, LCT, F-75005 Paris, France \\ ${ }^{\mathrm{b}}$ CNRS, UMR 7616, LCT, F-75005 Paris, France \\ ${ }^{\mathrm{c}}$ Department of Inorganic and Physical Chemistry, Ghent University, Krijgslaan 281 (S3), 9000 Gent, Belgium \\ ${ }^{\mathrm{d}}$ Laboratoire de Chimie Physique, UMR CNRS 8000, Groupe de Chimie Théorique, Université de Paris-Sud, 91405 Orsay Cédex, France
}

Keywords:

Valence-bond theory

Multiple bonding

Bonding analysis

\begin{abstract}
A B S T R A C T
An original procedure is proposed, based on valence bond theory, to calculate accurate dissociation energies for multiply bonded molecules, while always dealing with extremely compact wave functions involving three valence bond structures at most. The procedure consists of dividing the bond-breaking into sequential steps, thus breaking one by one the separate components of the multiple bond. By using the breathing-orbital valence bond method (Hiberty and Shaik, 2002), it is ensured that both static and dynamic differential electron correlations are taken into account in each step. The procedure is illustrated for typical examples of multiply bonded molecules, $\mathrm{N}_{2}, \mathrm{C}_{2}$ and $\mathrm{CO}$. The so-calculated total dissociation energies are at par with accurate calculations by state-of-the-art standard methods in the same basis set. The procedure also allows one to get some deep insight into the properties of the individual bonds that constitute the multiple bond. A so-called quasi-classical state is defined, in which the electrons of the bond under study have only one spin arrangement pattern, $\alpha \beta$, thus disabling the exchange of the two spin arrangements that is necessary for a covalent bonding interaction to take place. Taking this quasi-classical state as a non-bonded reference, one may estimate the "in-situ bonding energy" of an individual bond, as calculated at the molecular equilibrium geometry and in the presence of the other electrons. The procedure may also be used to assess the preferred bond length of an individual bond, which is shown to amount to $1.33 \AA$ for the $\sigma$ bond of $\mathrm{N}_{2}$, while the $\pi$ bonds get stronger and stronger as the interatomic distance is shortened. Another application is the calculation of the resonance energy arising from the mixing of the ionic components of an individual bond to its covalent component, and the comparison of this resonance energy with the in-situ bonding energy. This shows that the $\sigma$ bond of $N_{2}$ and $C_{2}$ is a classical covalent bond. On the other hand, the $\pi$ bonds have a substantial resonance energy that put them close to the category of charge-shift bonds.
\end{abstract}

\section{Introduction}

The calculation of accurate dissociation energies and dissociation energy profiles is perhaps the most challenging difficulty that any computational method has to face before being considered as fully reliable, and this is especially true in the case of multiple bonds. This is because both static and dynamic electron correlation energies are of crucial importance in the process of bond breaking. The problem becomes increasingly acute as a multiply bonded molecule is gradually stretched, as it quickly acquires strong multi-reference character. As an answer, a number of computational methods have been proposed during the last two decades,

* Corresponding authors at: Sorbonne Universités, UPMC Univ Paris 06, UMR 7616, LCT, F-75005 Paris, France (B. Braida).

E-mail addresses: benoit.braida@upmc.fr (B. Braida), philippe.hiberty@u-psud.fr (P.C. Hiberty) and most of them have been reviewed and evaluated in recent papers $[1,2]$. Leaving aside the vast majority of density functional methods, which are not strictly ab initio owing to their use of adjustable parameters, methods for computing wave functions can be of the single- or multi-reference type. The first type of methods is generally sufficient for calculating dissociation energies, by calculating the molecule at equilibrium geometries and the separate fragments, without dealing with the whole dissociation curve. There are however some exceptions, e.g., with the $C_{2}$ molecule which has significant multi-reference character, even at equilibrium [3]. Be it as it may, old methods like truncated configuration interaction $(\mathrm{CI})$ generally underestimate the dissociation energy, because they are not size-extensive if not improved by Davidson's correction [4] or by adding a quadratic term (e.g., QCISD [5]) to remedy for this defect. By contrast, the widely used Møller-Plesset perturbative expansion to second-order (MP2) is 
size-extensive and very economical, but generally overestimates dissociation energies [2].

The most widely used size-extensive single-reference methods are the coupled-cluster (CC) methods [6], which may include high-order excitations. In practice, the most commonly used CC methods are truncated at the single and double excitations level (CCSD) and may treat triple excitations by perturbation $(\operatorname{CCSD}(\mathrm{T}))$ [7]. Even better accuracy may be reached in a CCSD calculation when the effects of the single excitations are absorbed into the orbitals. The resulting orbitals are called Brueckner orbitals, and a determinant composed of such orbitals has the maximum possible overlap with the exact wave function. This type of calculation, which has the advantage of solving spurious symmetry-breaking problems, is called the Brueckner Doubles method (B-CCD) [8], and may also include additional perturbative estimate of the effects of triple excitations (B-CCD(T)) [9]. Other improvements of CC methods are the Renormalized- and Completely-Renormalized-CC methods $[10,11]$, in which one corrects the standard CC singles and doubles (CCSD) energy for the effects of triply and other higher-than-doubly excited clusters.

In case the single electronic configuration is not a qualitatively correct description of the system, or when one wishes to study stretched molecules or whole dissociation curves, one has to use multi-reference methods, in which the zeroth-order wave function involves several Slater determinants or configuration state functions. Many multi-reference methods use the Complete Active Space Self-Consistent Field (CASSCF) approach as a starting wave function, which takes the static electron correlation into account. The remaining correlation (dynamic correlation), may be obtained by further $\mathrm{CI}$, or by using perturbation theory, leading to the widely used CASPT2 approach [12]. A restricted active space may also be used, leading to the RASPT2 method. In the coupled-cluster family, multi-reference coupled-cluster (MRCC) methods [13] proved extremely successful, as well as their economical averaged-quadratic coupled-cluster (AQCC) variant [14], which starts from a CC ansatz and approximates those quadratic terms which are necessary to get nearly size-extensive results.

While being beautifully reliable and accurate, all the above methods have the inconvenience of displaying quite complicated wave functions expressed as very long expansions of configuration state functions, be the latter treated by $\mathrm{CI}$ of by perturbative treatments, hence yielding very little chemical insight. Thus, we have no information about the interplay of the individual bonds that constitute the multiple bond, neither on their specific properties: are they weak or strong, what are their preferred bond lengths, their degrees of covalency, what is the importance of alternative spin-couplings, and so on. What is needed to get this extra information is a simple method, yielding compact and easily interpretable though accurate wave functions. The breathing-orbital valence bond (BOVB) method [15] displays these latter features, as describing any two-electron bond as a combination of only three VB structures, while including not only static correlation, but also the necessary dynamic correlation. Despite this compactness of the wave functions, the method has long been shown to provide accurate bonding energies [16,17], together with unique bond properties like coefficients and weights of the covalent and ionic VB structures, as well as resonance energies arising from their mixing. However, the BOVB method, in its original formulation, becomes more complicated in case of multiple bonding, because the total amount of VB structures that form a complete and non-redundant basis set rapidly increases with the number of orbitals and electrons. For example, a triple bond, which is a 6-electron/6-orbital system, requires as much as $175 \mathrm{VB}$ structures, if all covalent and ionic structures are taken into account. Therefore, an alternative strategy has to be devised in order to get detailed information on the different components of multiple bonds, while still dealing with simple wave functions. The aim of the present work is to take up this challenge, and to devise an original method which will be applied to typical multiply bonded molecules, namely $\mathrm{N}_{2}, \mathrm{C}_{2}$, and $\mathrm{CO}$.

\section{Computational methods}

A many-electron system wave function, $\Psi$, is expressed in VB theory as a linear combination of Heitler-London-Slater-Pauling functions, $\Phi_{\mathrm{K}}$ in Eq. (1),

$\Psi=\sum_{\mathrm{K}} C_{\mathrm{K}} \Phi_{\mathrm{K}}$

where the $\Phi_{\mathrm{K}}$ functions correspond to 'classical' VB structures, and $C_{\mathrm{K}}$ are the corresponding structural coefficients.

The weights of the VB structures are calculated by means of the Coulson-Chirgwin formula [18], Eq. (2), which is the equivalent of a Mulliken population analysis in VB theory:

$W_{\mathrm{K}}=C_{\mathrm{K}}^{2}+\sum_{\mathrm{L} \neq \mathrm{K}} C_{\mathrm{K}} C_{\mathrm{L}}\left\langle\Phi_{\mathrm{K}} \mid \Phi_{\mathrm{L}}\right\rangle$

Here $\left\langle\Phi_{\mathrm{k}} \mid \Phi_{\mathrm{L}}\right\rangle$ is the overlap integral of two VB structures.

The most basic ab initio VB procedure is the VBSCF method [19], where the coefficients of both the orbitals determining the VB structures and structural coefficients themselves are variationally optimized simultaneously. This method takes static electron correlation into account. Dynamical correlation, however, needs to be included by a higher level method. One of these methods is the breathing-orbital valence bond method (BOVB) [15] that improves the VBSCF wave function without increasing the number of VB structures $\Phi_{\mathrm{K}}$, by allowing each VB structure to have its specific set of orbitals, different from one VB structure to the other, during the optimization process. In this manner, the orbitals can fluctuate in size and shape so as to fit the instantaneous charges of the atoms on which these orbitals are located, and this has long been shown to bring in the differential dynamic correlation of a bond alongside a breaking process, i.e., that part of the total dynamic correlation that varies from the reactants to the products of the bond-breaking reaction [15]. In this work, both VBSCF and BOVB calculations use atomic orbitals (AOs) that remain purely localized on a unique center, so as to ensure a perfectly clear correspondence between the mathematical expressions of the VB structures and their physical meaning, ionic or covalent.

While the bond that is broken in a given bond-breaking reaction will be described at the BOVB level in this paper, the remaining bonds, that keep unchanged alongside the reaction, will be described at the generalized valence bond (GVB) level [20], which uses semi-localized orbitals. With this method, the wave function for a two-electron bond is a formally covalent singlet-coupling between two orbitals $\phi_{\mathrm{L}}$ and $\phi_{\mathrm{R}}$, which are optimized with freedom to delocalize over the two centers, as in Eq. (3):

$\Psi^{\mathrm{GVB}}=\left|\phi_{\mathrm{L}} \bar{\phi}_{\mathrm{R}}\right|-\left|\bar{\phi}_{\mathrm{L}} \phi_{\mathrm{R}}\right|$

$\phi_{\mathrm{L}}=\varphi_{\mathrm{a}}+\varepsilon \varphi_{\mathrm{b}}$

$\phi_{\mathrm{R}}=\varphi_{\mathrm{b}}+\varepsilon \varphi_{\mathrm{a}}$

where the normalization factors have been dropped, and $\varphi_{a}$ and $\varphi_{b}$ are purely localized orbitals. It has been shown that this description is equivalent to a classical description displaying explicit covalent and ionic structures defined with purely localized orbitals [21,22]. For a multiple bond, when a unique VB structure is allowed (for example the triply-bonded structure $\mathbf{1}$ below), the GVB method is used in the so-called perfect-pairing approximation, GVB-PP. Both VBSCF and GVB calculations provide static correlation energy, but not dynamic correlation. 
The VB calculations have been carried out with the Xiamen Valence Bond (XMVB) program, available within the GAMESS package. High-level reference calculations by standard methods in the molecular-orbital framework of MO theory were made by means of the Gaussian09 package [23] or taken from other authors. The correlation-consistent triple-zeta (cc-pVTZ) basis sets of Dunning and coworkers [24] was used.

\section{Results and discussions}

\subsection{A strategy to probe the properties of individual components of multiple bonding}

In multiple bonds, like in $\mathrm{N}_{2}$ which will be used here to illustrate the method, it is not simple to experimentally determine separate bond energies and other properties for $\sigma$ and $\pi$ bonds. In double bonds (e.g., ethylene), one can roughly estimate the $\pi$ bonding energy as the rotational barrier, however this quantity also involves relaxation of the $\sigma$ bond and hyperconjugation in the twisted form. The problem gets even worse with triple bonds, since these species do not have rotational barriers. However, as we have amply shown before [25], these difficulties can be bypassed by defining a non-bonded reference state for one particular bond ( $\pi$ or $\sigma$ ), in which the two electrons maintain opposite spins but do not exchange. For example, if one wants to estimate the strength of one of the two $\pi$ bonds in the triply bonded structure of $\mathrm{N}_{2}$, say the $\pi_{\mathrm{x}}$ bond in the $x z$ plane, the non-bonded state, socalled "quasi-classical state" $\Psi_{\mathrm{QC}}^{\pi \mathrm{x}}$ in Scheme 1 , is a function in which the electrons of the $\pi_{\mathrm{x}}$ bond have only one spin arrangement pattern (only $\alpha \beta$ ). Since the bonding energy in a covalent two-electron bond is essentially due to the exchange of the two spin arrangements $(\alpha \beta \leftrightarrow \beta \alpha)$, the quasi-classical state by itself has no bonding and can be taken as a non-bonded reference. Indeed, in such a state, the interactions across the unpaired $\pi$ bond in $\Psi_{\mathrm{OC}}^{\pi \mathrm{x}}$ involve only classical electron-electron repulsion, nuclear-repulsion and electron-nuclear attraction, and since the fragments are neutral, these terms sum to approximately zero. By contrast, the wave function $\Psi_{\text {full }}^{\pi \mathrm{x}}$, which involves the three VB structures that are necessary to describe the $\pi_{\mathrm{x}}$ bond under study (one covalent and two ionic ones, as shown in Scheme 1) displays a fully bonding $\pi$ interaction. Thus, the difference between the energy of $\Psi_{\mathrm{QC}}^{\pi_{\mathrm{X}}}$ and

(a)

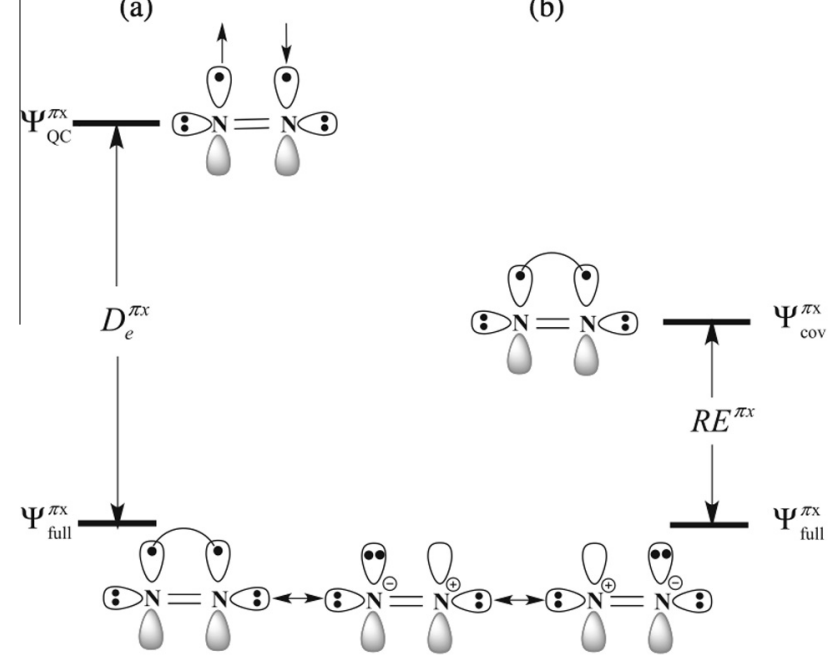

Scheme 1. (a) Definition of the quasi-classical state corresponding to the breaking of the $\pi_{\mathrm{x}}$ bond in $N_{2}$. (b) Definition of the charge-shift resonance energy arising from the mixing purely covalent with ionic structures for the $\pi_{\mathrm{x}}$ bond. that of $\Psi_{\text {full }}^{\pi_{\mathrm{x}}}$ gives the in-situ bonding energy $D_{\mathrm{e}}^{\pi \mathrm{x}}$ of the $\pi_{\mathrm{x}}$ bond at a given interatomic distance, i.e., the bonding energy that effectively stabilizes the electronic interaction of the $\pi_{\mathrm{x}}$ bond without any other relaxation or reorganization term arising from other bonds. The in-situ $\pi$-bonding energy is expressed in Eq. (4):

$D_{\mathrm{e}}^{\pi \mathrm{x}}=E\left(\Psi_{\mathrm{QC}}^{\pi \mathrm{x}}\right)-E\left(\Psi_{\text {full }}^{\pi \mathrm{x}}\right)$

where the remaining $\sigma$ and $\pi_{\mathrm{y}}$ bonds are treated at the GVB level (see Section 2), while the lone pairs are simple doubly occupied MOs. It is also easy to define a function $\Psi_{\text {cov }}^{\pi \mathrm{x}}$ in which the $\pi_{\mathrm{x}}$ bond is reduced to its covalent component, as illustrated in Scheme 1 where the curved line connecting the two electrons signifies the combination of the two spin arrangements, which is the very definition of a purely covalent bond. Here, the difference between the energy of $\Psi_{\text {cov }}^{\pi \mathrm{x}}$ and that of $\Psi_{\text {full }}^{\pi \mathrm{x}}$ gives the so-called "charge-shift" resonance energy $R E^{\pi x}$ arising from the mixing of the ionic structures with the covalent one [26]:

$\mathrm{RE}^{\pi \mathrm{x}}=E\left(\Psi_{\text {cov }}^{\pi \mathrm{x}}\right)-E\left(\Psi_{\text {full }}^{\pi \mathrm{x}}\right)$

Of course, analogous definitions will be used for probing the properties of the $\sigma$ bond, Eqs. (6) and (7):

$D_{\mathrm{e}}^{\sigma}=E\left(\Psi_{\mathrm{QC}}^{\sigma}\right)-E\left(\Psi_{\text {full }}^{\sigma}\right)$

$\mathrm{RE}^{\sigma}=E\left(\Psi_{\text {cov }}^{\sigma}\right)-E\left(\Psi_{\text {full }}^{\sigma}\right)$

where this time, the $\sigma$ bond is described at the BOVB level, while the other electrons are treated at a simpler level (vide infra).

The above strategy and definitions can be used to get some specific insight and information that standard methods cannot provide: (i) assessment of binding energy of each individual bond that contributes to the multiple bond. Incidentally, in-situ $\pi$ bonding energies have been calculated in the past [27] for a series of doubly-bonded molecules using Eq. (4), and were found to display regular and logical tendencies across the periodic table (bond strengthening upon moving from left to right). This was not the case with $\pi$ bonding energies estimated in a standard way as rotational barriers, (ii) assessment of the preferred bond length of each individual bond, obtained by calculating $D_{\mathrm{e}}^{\pi}$ and $D_{\mathrm{e}}^{\sigma}$ as functions of interatomic distances, (iii) respective coefficients and weights of the covalent and ionic components of the individual bonds, obtained from the VB expressions of their wave functions $\Psi_{\text {full }}^{\pi}$ and $\Psi_{\text {full }}^{\sigma}$, and (iv) for each individual bond, calculation of the resonance energy arising from the mixing of ionic structures with the covalent one, by means of Eqs. (5) and (7). Depending on its magnitude, this quantity will qualify the bond under study as a classical covalent bond (small RE) or as a charge-shift bond [26] (large RE).

So defined, the in-situ bonding energies provide some information that cannot be obtained from experiment. However, they can be inserted in a thermodynamic cycle, Eqs. (8)-(14), which eventually yields the total bonding energy of the triple bond, which can be compared with experiment or state-of-the-art calculations. The principle of the stepwise calculation of a multiple bond dissociation energy is very simple and consists of breaking the bonds one by one, decoupling the electron pair to a triplet, as in the series: $\mathrm{N} \equiv \mathrm{N} \rightarrow \mathrm{N}^{\uparrow}={ }^{\uparrow} \mathrm{N} \rightarrow \mathrm{N}^{\uparrow \uparrow}-{ }^{\uparrow \uparrow} \mathrm{N} \rightarrow \mathrm{N}^{\uparrow \uparrow \uparrow \uparrow \uparrow} \mathrm{N} \rightarrow 2 \mathrm{~N}^{\uparrow \uparrow \uparrow}$ If the in-situ bonding energies are to be inserted in the process, one gets the following steps:

$$
\begin{aligned}
& \mathrm{N} \equiv \mathrm{N} \rightarrow \mathrm{N}^{\uparrow}={ }^{\downarrow} \mathrm{N} \quad \Delta E_{1}=D_{\mathrm{e}}^{\pi \mathrm{x}} \\
& \mathrm{N}^{\uparrow}={ }^{\downarrow} \mathrm{N} \rightarrow \mathrm{N}^{\uparrow}={ }^{\uparrow} \mathrm{N} \quad \Delta E_{2} \\
& \mathrm{~N}^{\uparrow}={ }^{\uparrow} \mathrm{N} \rightarrow \mathrm{N}^{\uparrow \uparrow}={ }^{\downarrow} \mathrm{N} \quad \Delta E_{3}=D_{\mathrm{e}}^{\pi \mathrm{y}}
\end{aligned}
$$


$\mathrm{N}^{\Uparrow \uparrow}-{ }^{\imath \uparrow} \mathrm{N} \rightarrow \mathrm{N}^{\uparrow \uparrow}-{ }^{\uparrow \uparrow} \mathrm{N} \quad \Delta E_{4}$

$\mathrm{N}^{\uparrow \uparrow}-{ }^{\uparrow} \mathrm{N} \rightarrow \mathrm{N}^{\uparrow \uparrow \downarrow \uparrow \uparrow} \mathrm{N} \quad \Delta E_{5}=D_{e}^{\sigma}$

$\mathrm{N}^{\Uparrow \uparrow \uparrow \uparrow \uparrow} \mathrm{N} \rightarrow \mathrm{N}^{\Uparrow \uparrow \uparrow \uparrow \uparrow} \mathrm{N} \quad \Delta E_{6}$

$\mathrm{N}^{\Uparrow \uparrow \uparrow \uparrow \uparrow} \mathrm{N} \rightarrow \mathrm{N}^{\Uparrow \uparrow}+{ }^{\Uparrow \uparrow} \mathrm{N} \quad \Delta E_{7}$

Here Eq. (8) gives the in-situ bonding energy of the $\pi_{\mathrm{x}}$ bond, of which the electron pair is further spin-flipped in Eq. (9) to give a triplet state, and the process is repeated for the $\pi_{\mathrm{y}}$ bond (Eqs. (10) and (11)) and for the $\sigma$ bond (Eqs. (12) and (13)). The complete cycle is performed at the equilibrium geometry of the triply-bonded molecule. Finally, the geometry of the septet state, where all bonds have been uncoupled to parallel spins, is relaxed to give the two separate fragments, each in their ground quadruplet state. It is clear that the sum of reaction energies $\Delta E_{\mathrm{i}}(i=1-7)$ is equal to the total triple bond dissociation energy, regardless of the level of calculation that is used. Now, it is well known that accurately calculating multiple bond energies is challenging, owing to the necessity of taking both static and dynamic electron correlations into account, which justifies all the elaborate computational methods that have been devised (see above). Yet, the decomposition of the dissociation process as Eqs. (8)-(14) and the use of the BOVB method make the above requirement an easy task by allowing one to accurately taking into account the correlation energy effects for each step of the overall dissociation, while dealing with wave functions never larger than 3-configuration expansions, as is detailed below.

Let us consider the breaking of the $\pi_{\mathrm{x}}$ bond, and decoupling of its two electrons to quasi-classical state (Eq. (8)) then to a triplet (Eq. (9)). In the triply bonded structure, $\mathrm{N} \equiv \mathrm{N}$, the $\pi_{\mathrm{x}}$ bond is described as three VB structures, one covalent and two ionic ones, as shown in Scheme 1. On the other hand, the lone pairs are left uncorrelated and described at the simple MO level, whereas the remaining bonds ( $\pi_{\mathrm{y}}$ and $\sigma$ ) are described at the GVB level, up to the product of Eq. (9), $\mathrm{N}^{\uparrow}={ }^{\uparrow} \mathrm{N}$. Thus, static electron correlation is ensured for all bonds: (i) for the $\pi_{\mathrm{x}}$ bond, by means of the 3-structure description with simultaneous optimization of the coefficients and orbitals of the VB structures and (ii) by the GVB description for the remaining bonds. Furthermore, the dynamic correlation associated with the breaking of the $\pi_{\mathrm{x}}$ bond under study is also ensured by using the BOVB method [15], which allows each of the three VB structures to have its specific set of orbitals, different from one structure to the other (see Section 2). Thus, even if this method certainly does not provide the whole correlation energy for the molecule, still the differential correlation energy that is provided is all what is needed to yield an accurate value for $\Delta E_{1}+\Delta E_{2}$, which measures the energy cost of decoupling the $\pi_{\mathrm{x}}$ electron pair to a triplet.

The next steps are calculated in an analogous way. That is, for Eqs. (10) and (11), the $\pi_{\mathrm{y}}$ bond in $\mathrm{N}^{\uparrow}={ }^{\uparrow} \mathrm{N}$ is described as three VB structures, while the lone pair electrons and the $\sigma$ bond are treated at the simple MO and GVB levels, respectively, up to the product of Eq. (11), $\mathrm{N}^{\uparrow \uparrow}-{ }^{\uparrow} \mathrm{N}$. Two points are noteworthy: (1) the species $N^{\uparrow}={ }^{\uparrow} N$ is described differently depending on whether it is the product of reaction (9) (the $\pi_{\mathrm{y}}$ and $\sigma$ bonds are described at the GVB level) or the reactant of reaction (10) $\left(\pi_{\mathrm{y}}\right.$ is now described at the BOVB level, with one covalent and two ionic classical VB structures); actually this is the key that allows an easy calculation of differential correlation energy for each step. (2) The calculations of the in-situ bonding energies of $\pi_{\mathrm{x}}$ and $\pi_{\mathrm{y}}$ are not strictly equivalent, as $\pi_{\mathrm{x}}$ is surrounded by two bonds in Eq. (8) while $\pi_{\mathrm{y}}$ is surrounded by only one bond and a triplet pair of electrons in Eq. (10). Thus, we expect slightly different values for these two quantities.

The breaking of the $\sigma$ bond, Eqs. (12) and (13), is done in the same spirit. Finally, Eq. (14) is the relaxation of the septuplet species $\mathrm{N}^{\uparrow \uparrow \uparrow \uparrow \uparrow} \mathrm{N}$, in which all bonds are decoupled, to two separate ${ }^{\top \uparrow \uparrow} \mathrm{N}$ atoms in their quadruplet states. The final multiple bonding energy of the triply bonded structure is given by summing the reaction energies of Eqs. (8)-(14). Now, a final correction still has to be made, due to the fact that for a multiply bonded molecule like $\mathrm{N}_{2}$, making a triple bond (one $\sigma$ and two $\pi$ ones) is not the only way of singlet-coupling electrons in a 6-orbital/6-electron systems. There are in fact five possible spin-coupling schemes, structures 1-5 in Scheme 2. Thus, a correction for multiple spin-coupling shall be included, and can be computed by taking the energy difference between a wave function corresponding to structure $\mathbf{1}$ alone and a wave function corresponding to the mixing of 1-5. In this particular calculation, the orbitals are of the bond-distorted type so as to implicitly include ionic structures (Eqs. (3a)-(3c)), as is done in generalized valence bond (GVB) [20] or spin-coupled [28] theories. Of course, structure $\mathbf{1}$ is by far the major one at equilibrium distance, but structures 2-5 make a non-negligible energetic contribution. The final dissociation energy for $\mathrm{N}_{2}$, or any other triply bonded molecule, is obtained by adding the correction $\Delta E_{\mathrm{MR}}$ for multi-reference character (Eq. (15) below) to the sum of energies $\Delta E_{\mathrm{i}}$ arising from Eqs. (8)-(14):

$D_{\mathrm{e}}=\sum_{\mathrm{i}=1}^{\mathrm{i}=7} \Delta E_{\mathrm{i}}+\Delta E_{\mathrm{MR}}$

$\Delta E_{\mathrm{MR}}=E^{\mathrm{GVB}}(1)-E^{\mathrm{GVB}}(1-5)$

where the two terms in the right-hand side of Eq. (15b) represent the GVB energies of structure $\mathbf{1}$ alone and of the combination of structures 1-5, respectively.

\subsection{How adequate is the perfect-pairing representation of a multiply-} bonded molecule? The $\mathrm{N}_{2}$ example

Since a 6-electron/6-orbital system like that of $\mathrm{N}_{2}$ involves five possible linearly independent coupling schemes, one may wonder whether or not the triply-bonded structure ( $\mathbf{1}$ in Scheme 2 ) is an adequate representation of this molecule at equilibrium distance, and what is the importance of the other VB structures as one stretches the molecule. This can be easily tested by performing a full GVB calculation on this molecule, involving the five coupling schemes 1-5, and calculating their weights at various interatomic distances using Eq. (2).

The results are displayed in Fig. 1. It appears that at equilibrium distance (1.1033 Å at the MP2/cc-pVTZ level) and shorter, the perfect-pairing structure $\mathbf{1}$ is by far the most important one, with a weight of ca. $75 \%$, followed by structures $2-4$ with weights of about $8 \%$ each. Finally, structure $\mathbf{5}$ is completely negligible at all distances. These results are quite logical since structure $\mathbf{1}$ is the most stable one as it displays three bonds, followed by 2-4 which each display one single bond, either of $\pi$ or $\sigma$ type, whereas structure 5 does not display any bond. Now, this ordering is necessarily reshuffled as the molecule is stretched since eventually the electronic state must correspond to two $\mathrm{N}$ atoms each in a quadruplet state, an overall electronic state quite different from structure $\mathbf{1}$ alone in the super-system. Indeed, as the interatomic distance increases, structures $\mathbf{2}$ and $\mathbf{3}$ increase their weights, structure $\mathbf{4}$ increases its weight even more sharply, whereas the weight of structure $\mathbf{1}$ collapses, to cross the curve of structure $\mathbf{4}$ and eventually that of $\mathbf{2}$ and $\mathbf{3}$. The variations of the weights for structures $\mathbf{4} \mathrm{vs}$ 2,3 are easily explained by the nature of the single bonds in these respective VB structures. In structure 4 , the single bond is of $\sigma$ type, and is therefore in a geometry shorter than optimal (as will be confirmed in the next section) due to the compressing effect of the $\pi$ bonds at the equilibrium distance of $\mathrm{N}_{2}$. Thus, stretching the molecule from equilibrium distance has, at least at the 


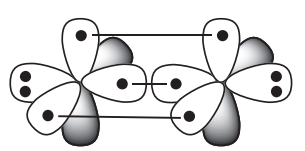

1

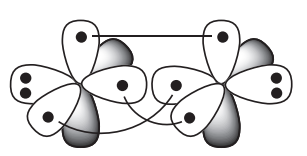

2

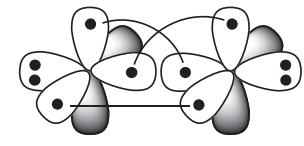

3

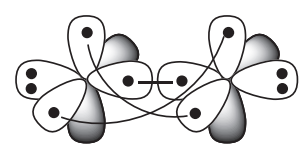

4

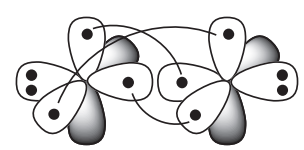

5

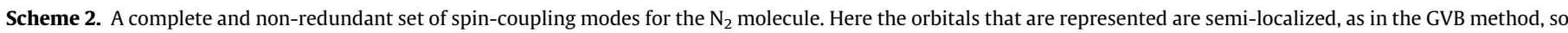
that the formally covalent VB structures implicitly include the ionic ones.

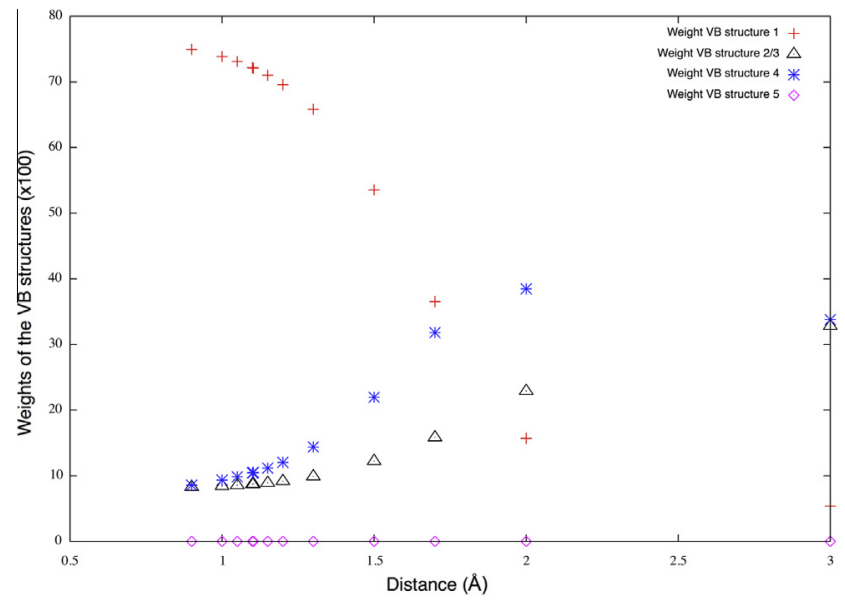

Fig. 1. Weights of structures $\mathbf{1 - 5}$ in $\mathrm{N}_{2}$ as a function of the interatomic distance.

beginning, the effect of reinforcing the $\sigma$ bond and the weight of 4 . On the other hand, structures $\mathbf{2}$ and $\mathbf{3}$ each display a single $\pi$ bond, which is destabilized by stretching. As a consequence, the difference in weights of $\mathbf{2 / 3}$ and $\mathbf{4}$ is increasing as the interatomic distance is stretched.

Another indication of the importance or non-importance of structures 2-5 is the calculation of the correction for multireference character, $\Delta E_{\mathrm{MR}}$ in Eq. (15b), as a function of the interatomic distance. The result is shown in Fig. 2 for the $\mathrm{N}_{2}$ molecule. At

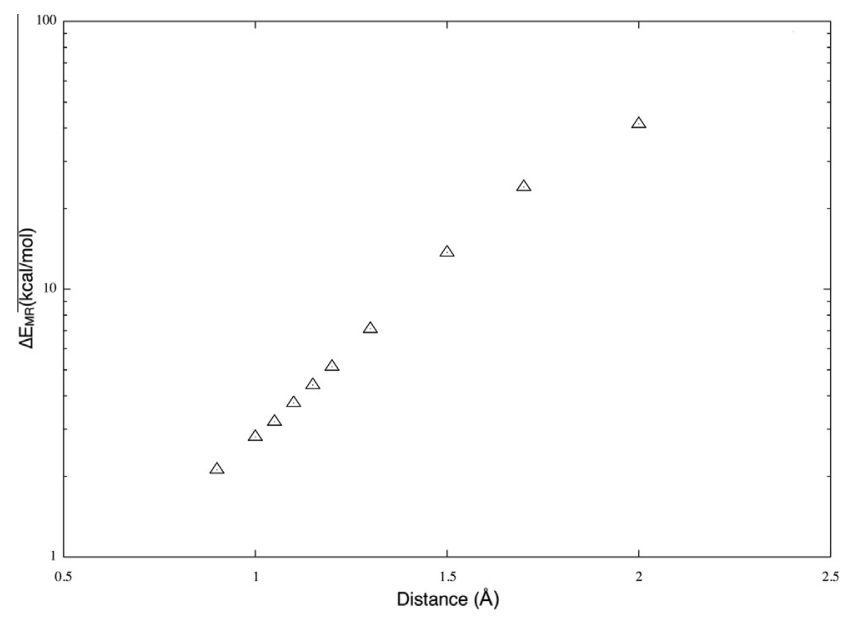

Fig. 2. The correction $\Delta E_{\mathrm{MR}}$ for secondary spin-couplings of $\mathrm{N}_{2}$ as a function of the interatomic distance. equilibrium distance, the stabilization brought by the mixing of 2-5 with $\mathbf{1}$, as measured by $\Delta E_{\mathrm{MR}}$, is rather low, less than $4 \mathrm{kcal} /$ mol. However, this term increases rather sharply as the interatomic distance is stretched, as shown by a quasi-linear curve as plotted with a semi-logarithmic scale, to reach ca. $40 \mathrm{kcal} / \mathrm{mol}$ at a distance of $2 \AA$. Thus, Figs. 1 and 2 both lead to the conclusion that the perfect-pairing approximation, on which the calculation of the total bonding energy is based, is excellent at equilibrium distance and only necessitates a small $\Delta E_{\mathrm{MR}}$ correction. On the other hand, as the correction quickly increases as the molecule is stretched, our thermodynamic cycle in Eqs. (8)-(13) could not be used to calculate a full dissociation energy curve by Eq. (15). However, this restriction does not apply to the study of individual bond energy curves in the next section.

\subsection{A one-by-one study of the individual bonds}

Let us now consider one of the two $\pi$ bonds, say $\pi_{x}$, the one involving AOs lying in the $z x$ plane. For this bond, a quasi-classical state $\Psi_{\mathrm{QC}}^{\pi \mathrm{x}}$ is defined as the non-bonded reference, whose difference in energy with respect to the fully bonding wave function $\Psi_{\text {full }}^{\pi \mathrm{x}}$ defines the in-situ bonding energy (see Eq. (4) and Scheme 1). With these definitions, one may calculate the bonding energy of the $\pi_{\mathrm{x}}$ bond alone, without altering the other bonds and lone pairs of the molecule under study, and such a point-by-point calculation at various distances allows one to calculate the dissociation energy curve of this particular bond, following the bond-breaking process expressed by Eq. (8).

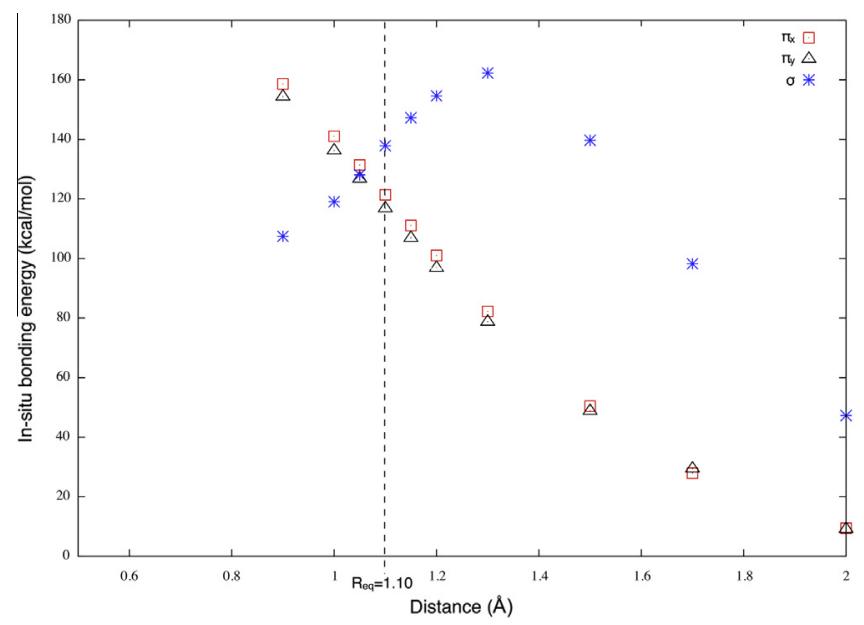

Fig. 3. In-situ bonding energies of the $\pi_{x}, \pi_{y}$ and $\sigma$ bonds of $N_{2}$ as a function of the interatomic distance. 
The dissociation energy curve of the $\pi_{\mathrm{x}}$ bond is plotted in Fig. 3 for the $\mathrm{N}_{2}$ molecule, with interatomic distances varying from 0.9 to $2.0 \AA$. It is seen that the bonding interaction is quite significant at the equilibrium distance of the molecule, ca. $122 \mathrm{kcal} / \mathrm{mol}$, and gets even stronger at shorter distances. On the other hand, it sharply decreases as the molecule is stretched, reaching a value as small as $8 \mathrm{kcal} / \mathrm{mol}$ at a distance of $2.0 \AA$. This preference of $\pi$ bonds for short distances was actually expected, as we have demonstrated in a previous paper [29] that there generally is a linear correlation between the in-situ bonding energies of $\pi$ bonds and the inverse of the squared interatomic bond lengths.

If studied in the same conditions as $\pi_{\mathrm{x}}$, the $\pi_{\mathrm{y}}$ bond would of course display exactly the same characteristics, since the two bonds are perfectly equivalent. It is however interesting to study the $\pi_{\mathrm{y}}$ bond according to Eq. (10), i.e., in the spirit of cutting the bonds one by one as in the thermodynamic cycle above (Eqs. (8)-(14)). Thus, we start from $\mathrm{N}^{\uparrow}={ }^{\uparrow} \mathrm{N}$, which displays a $\sigma$ bond and the $\pi_{\mathrm{y}}$ bond, whereas the electrons of the former $\pi_{\mathrm{x}}$ bond now have parallel spins. We then cut the $\pi_{\mathrm{y}}$ bond to form a quasi-classical state $\mathrm{N}^{\uparrow}-{ }^{\downarrow \uparrow} \mathrm{N}$ in which the electrons of the former $\pi_{\mathrm{y}}$ bond have opposite spins and are not allowed to exchange their positions. Thus, the difference between Eqs. (8) and (10) lies in the environment of the bond under study: two lone pairs and two bonds for $\pi_{\mathrm{x}}$ (Eq. (8)), vs two lone pairs, one bond and two triplet-coupled $\pi$ electrons for $\pi_{\mathrm{y}}$ (Eq. (10)). Still, despite this difference, the dissociation energy curve of $\pi_{\mathrm{y}}$ closely matches that of $\pi_{\mathrm{x}}$ in Fig. 3 .

The study of the $\sigma$ bond follows the same line, Eq. (12). Here, the bond under study is surrounded by two lone pairs, and four electrons having parallel spins, corresponding to the former $\pi_{\mathrm{x}}$ and $\pi_{\mathrm{y}}$ bonds. The dissociation energy curve of this individual bond is most interesting, as it displays a maximum of bond strength for a distance of $1.33 \AA$, some $0.23 \AA$ longer than the equilibrium distance of $\mathrm{N}_{2}$. This distance is shorter than the single $\mathrm{N}-\mathrm{N} \sigma$ bond in hydrazine (1.446 $\AA$ ), which is normal owing to the different hybridization of the orbitals implied in the $\sigma$ bond, $s p^{3}$ in hydrazine vs $s p$ in $\mathrm{N}_{2}$ (it is known that $s p-s p$ bonds are shorter and stronger than $s p^{3}-s p^{3}$ bonds). Be it as it may, this clearly shows that the $\sigma$ bond in $\mathrm{N}_{2}$ (and of course presumably in all multiply-bonded molecules) is a compressed one, with a preferred bond length quantified by VB theory.

The in-situ bonding energies are given in Table 1 for $\mathrm{N}_{2}$ in its equilibrium geometry, showing that the $\sigma$ bond is stronger than the $\pi$ ones. Another quantity of interest, for each individual bond, is the resonance energy arising from the mixing of the ionic components to the major covalent one (Scheme 1b and Eqs. (5) and (7)). It is seen that this resonance energy is quite moderate for the $\sigma$ bond, $23.4 \mathrm{kcal} / \mathrm{mol}$, which represents ca. $17 \%$ of the full in-situ bonding energy, which is the range of classical covalent bonds [26]. On the other hand, the covalent-ionic resonance energies amount to significantly larger values for the $\pi$ bonds, ca. $50 \mathrm{kcal} / \mathrm{mol}$ in average, i.e., about $42 \%$ of a total in-situ bonding energy of $\sim 120 \mathrm{kcal} / \mathrm{mol}$. Such values, albeit being too small to classify the $\pi$ bonds of $\mathrm{N}_{2}$ as charge-shift bonds, still show that these $\pi$ bonds have an important charge-shift character, as was already found in doubly-bonded species in a former study [27].

Table 1

Properties of the individual bonds of the $\mathrm{N}_{2}$ molecule, at an MP2-optimized interatomic distance of $1.1033 \AA$ A . Energies in $\mathrm{kcal} / \mathrm{mol}$.

\begin{tabular}{llll}
\hline & $\Delta E\left(\Psi_{\text {full }} \rightarrow \Psi_{\mathrm{QC}}\right)$ & $\mathrm{RE}^{\mathrm{a}}$ & CS factor $(\%)^{\mathrm{b}}$ \\
\hline$\pi_{\mathrm{x}}$ & 122.4 & 54.3 & 44.3 \\
$\pi_{\mathrm{y}}$ & 118.0 & 47.8 & 40.5 \\
$\sigma$ & 139.0 & 23.4 & 16.8 \\
\hline
\end{tabular}

a Defined as in Eq. (5) for the $\pi$ bonds, and as in Eq. (7) for the $\sigma$ bond.

b Charge-shift factor, defined as $\operatorname{RE} / \Delta E\left(\Psi_{\text {full }} \rightarrow \Psi_{\text {QC }}\right)$.
The same kind of study has been performed in this work for the $\mathrm{C}_{2}$ molecule. As shown by some recent studies at a rather high level of VB theory [30], the major VB structure for this molecule involves two $\pi$ bonds and one inner $\sigma$ bond, just like in $\mathrm{N}_{2}$, plus a fourth weak outer bond between the external hybrid AOs pointing away from the molecular center, as illustrated in Scheme 3.

Whether or not this fourth coupling is strong enough to be considered as a bond has stirred some controversy [30c-f], which is not the subject of the present study. Be it as it may, the in-situ bonding energies of the $\pi$ bonds and inner $\sigma$ bond, displayed in Table 2, show some differences and resemblances as compared to those of $\mathrm{N}_{2}$. Indeed, the in-situ bonding energies are all significant for these three bonds, but consistently smaller than the corresponding ones in $\mathrm{N}_{2}$, which is easily understandable by the fact that $\mathrm{C}$ is situated on the left-hand side of $\mathrm{N}$ in the periodic table. On the other hand, the charge-shift characters of these individual bonds are exactly the same as those of $\mathrm{N}_{2}$, classifying the inner $\sigma$ bond as classically covalent and the $\pi$ ones as close to charge-shift bonds. Finally, the outer $\sigma$ bond has a small in-situ bonding energy, in agreement with previous studies [30a,b,e], and an extremely low charge-shift character, making this bond practically a (weak) purely covalent one.

\subsection{Calculation of the total multiple-bond dissociation energies}

The sequential breaking of individual bonds can also be used to calculate the total dissociation energy of a multiply-bonded molecule. For this purpose, the thermodynamic cycle above can be simplified, by directly uncoupling a given bond to a triplet (cutting the weakest bonds first), thus by-passing the quasi-classical state. Thus, the process is reduced to the four steps below:

$\mathrm{N} \equiv \mathrm{N} \rightarrow \mathrm{N}^{\uparrow} \equiv{ }^{\uparrow} \mathrm{N} \quad \Delta E_{1}+\Delta E_{2}$

$\mathrm{N}^{\uparrow} \equiv{ }^{\uparrow} \mathrm{N} \rightarrow \mathrm{N}^{\uparrow \uparrow}-{ }^{\uparrow} \mathrm{N} \quad \Delta E_{3}+\Delta E_{4}$

$\mathrm{N}^{\uparrow \uparrow}-{ }^{\uparrow} \mathrm{N} \rightarrow \mathrm{N}^{\uparrow \uparrow \uparrow \uparrow \uparrow} \mathrm{N} \quad \Delta E_{5}+\Delta E_{6}$

$\mathrm{N}^{\uparrow \uparrow \uparrow \uparrow \uparrow} \mathrm{N} \rightarrow \mathrm{N}^{\uparrow \uparrow \uparrow}+{ }^{\uparrow \uparrow} \mathrm{N} \quad \Delta E_{7}$

Eqs. (16)-(19) provide a way to calculate the total dissociation energy, a challenging spectroscopic constant, with extremely simple and compact wave functions, contrasting with the elaborate methods mentioned above. Another motivation for such calculations is the fact that the total dissociation energy is the outcome of the one-by-one bond-breaking, from which some quantitative information is extracted on the individual bonds. Thus, the accuracy of the total dissociation energies will be a test to check that the individual bonds are accurately described with only three VB structures, provided that an accurate VB method is used.

It is to be noted that, in Eqs. (16)-(19) as well as in Eqs. (8)-(14), an ordering has to be chosen for cutting the individual bonds one by one. Here we have chosen to cut the weakest bonds first (the $\pi$ ones), in order to keep the remaining underlying bonds as little perturbed as possible. The adequacy of this choice will be numerically tested below.

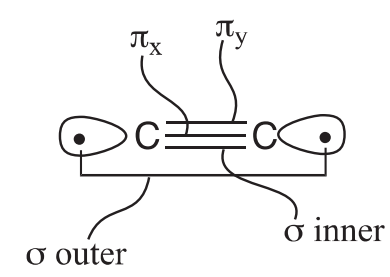

Scheme 3. The major VB structure for $C_{2}$. 
Table 2

Properties of the individual bonds of the $C_{2}$ molecule, at an MP2-optimized interatomic distance of $1.2139 \AA$ A . Energies in $\mathrm{kcal} / \mathrm{mol}$.

\begin{tabular}{lccc}
\hline & $\Delta E\left(\Psi_{\text {full }} \rightarrow \Psi_{\mathrm{QC}}\right)$ & $\mathrm{RE}^{\mathrm{a}}$ & CS factor $(\%)$ \\
\hline$\sigma_{\text {outer }}$ & 9.7 & 0.4 & 4.4 \\
$\pi_{\mathrm{x}}$ & 92.4 & 38.7 & 41.9 \\
$\pi_{\mathrm{y}}$ & 87.4 & 32.5 & 37.2 \\
$\sigma_{\text {inner }}$ & 72.1 & 10.9 & 15.1
\end{tabular}

a Defined as in Eq. (5) for the $\pi$ bonds, and as in Eq. (7) for the $\sigma$ bond.

The total dissociation energies are shown in Table 3, as calculated at various levels of VB theory, and compared to high-level standard calculations performed in the same cc-pVTZ basis set, and to experiment. The set of diatomic molecules that is used for this comparison has been chosen for the following reasons: $\mathrm{N}_{2}$ is the standard test-case for the calculation of triple bond dissociation energies, $C_{2}$ is a difficult test-case with known multi-reference character [3], and $\mathrm{CO}$ is a heteropolar analogue of $\mathrm{N}_{2}$.

To begin with, it can be seen that the dissociation energies calculated at the simple VBSCF level (column 1 in Table 3) are very far from the reference theoretical values and from experiment, with errors ranging from 40 to $65 \mathrm{kcal} / \mathrm{mol}$. This is obviously due to the fact that VBSCF lacks dynamic electron correlation, just like the CASSCF standard MO-based level, which is also known to yield very poor dissociation energies for multiplybonded molecules. On the other hand, allowing for dynamic correlation to be taken into account by going from VBSCF to the BOVB level brings a dramatic improvement. Even without the correction $\Delta E_{\mathrm{MR}}$ for multi-reference character, the mere BOVBcalculated dissociation energy for $\mathrm{N}_{2}$, ca. $212 \mathrm{kcal} / \mathrm{mol}$, is already quite close to the standard $\operatorname{CCSD}(\mathrm{T})$ value, ca. $216 \mathrm{kcal} / \mathrm{mol}$, showing that the perfect-pairing approximation (by which the molecule is described as a single VB structure ( $\mathbf{1}$ in Scheme 2)) is excellent. Adding the $\Delta E_{\mathrm{MR}}$ correction makes the BOVBcorrected and $\operatorname{CCSD}(\mathrm{T})$ values practically equivalent. On the other hand, calculating the total dissociation energy of $\mathrm{N}_{2}$ by cutting the bonds in a different order (the $\sigma$ bond first, then the $\pi$ ones), would yield a larger value, $228.7 \mathrm{kcal} / \mathrm{mol}$, practically equal to the experimental value. While this nice agreement might appear appealing, we believe that it is somewhat artificial, owing to the incompleteness of the basis set that we used. This confirms the soundness of our choice to cut the weakest bonds first in Eqs. (16)-(19), which yields a result at par with the best available calculations in the same basis set (Table 3 ).

In sharp contrast with $\mathrm{N}_{2}$, the perfect pairing approximation appears as unfounded for the $C_{2}$ molecule, for which the $\Delta E_{\mathrm{MR}}$ correction is as large as $21 \mathrm{kcal} / \mathrm{mol}$ at the equilibrium geometry. This is in harmony with our finding that the ground state of $C_{2}$ is best described as a combination of VB structures, among which the triply-bonded one (actually a quadruply bonded structure if one includes the weak $\sigma$-outer bond) is by far the major one, but not the only one [31]. This also confirms the known multi-reference character of this molecule, as found earlier in the framework of MO-based methods [3]. Nevertheless, the BOVB-corrected value

Table 3

Total dissociation energies $D_{\mathrm{e}}$ for some multiply bonded molecules, in $\mathrm{kcal} / \mathrm{mol}$.

\begin{tabular}{lrllll}
\hline & VBSCF & BOVB & BOVB $+\Delta E_{\mathrm{MR}}$ & Reference & Experimental \\
\hline $\mathrm{N}_{2}$ & 162.39 & 211.71 & 215.61 & $216.36^{\mathrm{a}}$ & $228.03^{\mathrm{b}}$ \\
$\mathrm{C}_{2}$ & 83.02 & 122.77 & 143.80 & $142.4^{\mathrm{c}}$ & $146.04^{\mathrm{b}}$ \\
$\mathrm{CO}$ & 198.85 & 254.19 & 256.90 & $248.54^{\mathrm{b}}$ & $259.26^{\mathrm{b}}$
\end{tabular}

\footnotetext{
a $\operatorname{CCSD}(\mathrm{T}) / \mathrm{cc}-\mathrm{pVTZ}$ calculation from Ref. [2]

b From Ref. [33].

c MCSCF full-valence CAS/CVB1 from Ref. [34]
}

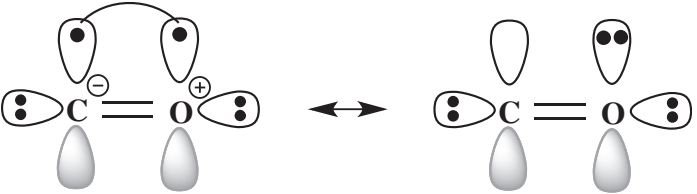

Scheme 4. BOVB representation of one of the $\pi$ bonds in carbon monoxide. The remaining VB structure, which would have the formal charge distribution $\mathrm{C}^{2-} \mathrm{O}^{2+}$, is not represented here.

for the dissociation energy of $C_{2}$ (third column) is excellent, in-between the experimental value and the reference theoretical value in a basis set of the same quality.

The perfect-pairing representation of carbon monoxide consists of a triple bond made of a $\sigma$ bond surrounded by two strongly polar $\pi$ bonds. In classical VB language, each $\pi$ bond can be represented as a combination of a covalent component and a significant ionic component, as shown in Scheme 4.

The perfect-pairing approximation appears to be excellent, as was found for $\mathrm{N}_{2}$, since the $\Delta E_{\mathrm{MR}}$ correction for multi-reference character amounts to slightly less than $3 \mathrm{kcal} / \mathrm{mol}$. Quite amazingly, the corrected BOVB value (third column) for the total dissociation energy appears to be significantly better than the reference $\operatorname{CCSD}(\mathrm{T})$ value, and close to the experimental value. BOVB-calculated bonding energies which lie in-between experimental values and best standard calculations in the same basis set are not unusual, and can be explained as follows. In the standard BOVB method, the "inactive" electrons, i.e., those electrons that belong to the bonds or lone pairs that are not treated at the VB level, are left uncorrelated, and lie in doubly-occupied orbitals (here the lone pairs). However, these "inactive" orbitals are different from one VB structure to the other, and this fact brings in a slight amount of correlation of the inactive electrons, which is not present in the dissociated products. This (small) artefact has the effect of slightly increasing the bonding energy, in a systematic way. On the other hand, using basis sets of medium size generally leads to underestimated bonding energies with respect to calculations in complete basis set. Thus, BOVB calculations in mediumsized basis sets are subject to two systematic errors which somehow compensate for each other, hence the good results that are often closer to experiment than even full $\mathrm{CI}$ in the same basis set [32]. In the CO case, the breathing-orbital effect is particularly large due to the importance of the ionic contributions in the $\pi$ bonds (see Scheme 4), as compared to the $\mathrm{N}_{2}$ case in which the covalent components are expected to be largely major.

\section{Conclusion and perspectives}

Whereas the calculation of dissociation energies for multiply bonded molecules generally requires state-of-the-art methods using long configuration expansions, the VB-based stepwise procedure that is proposed here only deals with extremely compact wave functions, involving three VB structures at most at each step. Despite this simplicity, the so-calculated dissociation energies prove accurate for the test-cases investigated here, and at par with best state-of-the-art method in the same basis set. The key to the combination of accuracy and simplicity is the use of the BOVB method for describing the individual bond that is broken in a given step, while the other bonds/lone pairs are described at a lower level. In this way, both static and dynamic differential electron correlations are taken into account in each step.

The procedure is also used to extract some information on the individual bonds that cannot be given by experiment. By defining a quasi-classical state that is taken as a non-bonded reference for a given individual bond, one estimates the in-situ bonding energy 
of this bond, which is calculated without having to resort to indirect methods such as rotational barriers and so on. The so-calculated bonding energy measures the energy arising from the bonding interaction of the individual bond under study, in the presence of the other electrons and at the molecular equilibrium geometry. Calculating such bonding energies at various distances allows one to assess the preferred bond length of, e.g., the $\sigma$ bond of a multiple bond, which amounts to $1.33 \AA$ in $\mathrm{N}_{2}$. By contrast, the $\pi$ bonds get stronger and stronger as the interatomic distance is shortened. Furthermore, by calculating covalent-ionic resonance energies for individual bonds, one estimates a charge-shift factor that is small in the $\sigma$ bonds of $N_{2}$ and $C_{2}$, but rather large in the $\pi$ bonds of these molecules. It is concluded that the $\sigma$ bond in a triple bond is a classical covalent bond, whereas the $\pi$ bonds are close to the category of charge-shift bonds.

Extension of this work to more difficult cases, like quadruply bonded molecules or even possibly sextuply bonded molecules like $\mathrm{Cr}_{2}, \mathrm{Mo}_{2}$ and $\mathrm{W}_{2}$ is envisaged.

\section{Acknowledgement}

Professor $\mathrm{W}$. Wu is gratefully acknowledged for making his ab initio VB program XMVB available to us.

\section{References}

[1] K.R. Yang, A. Jalan, W.H. Green, D.G. Truhlar, Which ab initio wave function methods are adequate for quantitative calculations of the energies of biradicals? the performance of coupled-cluster and multi-reference methods along a single-bond dissociation coordinate, J. Chem. Theory Comput. 9 (2013) 418-431.

[2] D. Robinson, Splitting multiple bonds: a comparison of methodologies on the accuracy of bond dissociation energies, J. Comput. Chem. 34 (2013) 26252634.

[3] J.D. Watts, J.D. Bartlett, Coupled-cluster calculations on the C2 molecule and the C2+ and C2- molecular ions, J. Chem. Phys. 96 (1992) 6073-6084.

[4] S.R. Langhoff, E.R. Davidson, Configuration interaction calculations on nitrogen molecule, Int. J. Quantum Chem. 8 (1974) 61-72.

[5] J.A. Pople, M. Head-Gordon, K. Raghavachari, Quadratic configuration interaction - a general technique for deterlining electron correlation energies, J. Chem. Phys. 87 (1987) 5968-5975.

[6] R.J. Bartlett, M. Musial, Coupled-cluster theory in quantum chemistry, Rev. Mod. Phys. 79 (2007) 291-352.

[7] K. Raghavachari, G.W. Trucks, J.A. Pople, M. Head-Gordon, A 5th-order perturbation comparison of electron correlation theories, Chem. Phys. Lett. 157 (1989) 479-483.

[8] R.A. Chiles, C.E. Dykstra, An electron pair operator approach to coupled cluster wave functions. application to $\mathrm{He}_{2}, \mathrm{Be}_{2}$, and $\mathrm{Mg}_{2}$ and comparison with CEPA methods, J. Chem. Phys. 74 (1981) 4544-4556.

[9] N.C. Handy, J.A. Pople, M. Head-Gordon, K. Raghavachari, G.W. Trucks, Sizeconsistent Brueckner theory limited to double substitutions, Chem. Phys. Lett. 164 (1989) 185-192.

[10] P. Piecuch, M. Wloch, AJC. Varandas, J.C. Antonio, Renormalized coupledcluster methods: theoretical foundations and application to the potential function of water, in: S. Lahmar, J. Maruani, S. Wilson, G. DelgadoBarrio (Eds.), Topics in the Theory of Chemical and Physical Systems, Springer, The Netherlands, 2007, pp. 63-121.

[11] K. Kowalski, P. Piecuch, The method of moments of coupled-cluster equations and the renormalized $\operatorname{CCSD}[\mathrm{T}], \operatorname{CCSD}(\mathrm{T}), \operatorname{CCSD}(\mathrm{TQ})$, and $\operatorname{CCSDT}(\mathrm{Q})$ approaches, J. Chem. Phys. 113 (2000) 18-35.

[12] K. Andersson, P.-Å. Malmqvist, B.O. Roos, 2nd-order perturbation theory with a complete active space self-consistent field reference function, J. Chem. Phys. 96 (1992) 1218-1226

[13] R.J. Bartlett, M. Musial, Coupled-cluster theory in quantum chemistry, Rev. Mod. Phys. 79 (2007) 291-352. and references therein.

[14] P.G. Szalay, R. Bartlett, Multi-reference averaged quadratic coupled-cluster method; a size-extensive modification of multi-reference $\mathrm{CI}$, Chem. Phys. Lett. 214 (1993) 481-488.

[15] (a) P.C. Hiberty, S. Humbel, C.P. Byrman, J.H. van Lenthe, Compact valence bond functions with breathing orbitals: application to the bond dissociation energies of F2 and FH, J. Chem. Phys. 101 (1994) 5969-5976;

(b) P.C. Hiberty, S. Shaik, BOVB-a modern valence bond method that includes dynamic correlation, Theor. Chem. Acc. 108 (2002) 255-272;

(c) P.C. Hiberty, S. Shaik, Breathing-orbital valence bond-a valence bond method incorporating static and dynamic electron correlation effects, in: D.L. Cooper (Ed.), Valence Bond Theory, Elsevier, Amsterdam, The Netherlands, 2002, pp. 187-226.
[16] (a) S. Shaik, D. Danovich, B. Silvi, D. Lauvergnat, P.C. Hiberty, Charge-shift bonding - a class of electron-pair bonds emerges from valence bond theory and supported by electron localization function approach, Chem. Eur. J. 11 (2005) 6358-6371.

[17] W. Wu, P. Su, S. Shaik, P.C. Hiberty, Classical valence bond approach by modern methods, Chem. Rev. 111 (2011) 7557-7593.

[18] H.B. Chirgwin, C.A. Coulson, The electronic structure of conjugated systems VI, Proc. R. Soc. London Ser. A 201 (1950) 196-209.

[19] (a) J.H. van Lenthe, G.G. Balint-Kurti, The valence-bond SCF (VB SCF) method. Synopsis of theory and test calculations of the $\mathrm{OH}$ potential energy curve, Chem. Phys. Lett. 76 (1980) 138-142;

(b) J.H. van Lenthe, G.G. Balint-Kurti, The valence-bond self-consistent field method (VB-SCF): theory and test calculations, J. Chem. Phys. 78 (1983) 56995713;

(c) J. Verbeek, J.H. van Lenthe, On the evaluation of nonorthogonal matrix elements, J. Mol. Struct.: THEOCHEM 229 (1991) 115-137.

[20] (a) F.W. Bobrowicz, W.A. Goddard III, The self-consistent field equations for generalized valence bond and open-shell Hartree-Fock wave functions, in: H.F. Schaefer (Ed.), Methods of Electronic Structure Theory, vol. 4, Plenum, New York, 1977, pp. 79-126;

(b) W.A. Goddard III, T.H. Dunning, W.J. Hunt, P.J. Hay, Generalized valence bond description of bonding in low-lying states of molecules, Acc. Chem. Res. 6 (1973) 368-376.

[21] S. Shaik, P.C. Hiberty, A Chemist's Guide to Valence Bond Theory, Wiley, Hoboken, 2008.

[22] S. Shaik, P.C. Hiberty, Valence bond theory, its history, fundamentals, and applications. A primer, Rev. Comput. Chem. 20 (2004) 1-100.

[23] M.J. Frisch, G.W. Trucks, H.B. Schlegel, G.E. Scuseria, M.A. Robb, J.R. Cheeseman, G. Scalmani, V. Barone, B. Mennucci, G.A. Petersson, H. Nakatsuji, M. Caricato, X. Li, H.P. Hratchian, A.F. Izmaylov, J. Bloino, G. Zheng, J.L. Sonnenberg, M. Hada, M. Ehara, K. Toyota, R. Fukuda, J. Hasegawa, M. Ishida, T. Nakajima, Y. Honda, O. Kitao, H. Nakai, T. Vreven, J.A. Montgomery, Jr., J.E. Peralta, F. Ogliaro, M. Bearpark, J.J. Heyd, E. Brothers, K.N. Kudin, V.N. Staroverov, R. Kobayashi, J. Normand, K. Raghavachari, A. Rendell, J.C. Burant, S.S. Iyengar, J. Tomasi, M. Cossi, N. Rega, J.M. Millam, M. Klene, J.E. Knox, J.B. Cross, V. Bakken, C. Adamo, J. Jaramillo, R. Gomperts, R.E. Stratmann, O. Yazyev, A.J. Austin, R. Cammi, C. Pomelli, J.W. Ochterski, R.L. Martin, K. Morokuma, V.G. Zakrzewski, G.A. Voth, P. Salvador, J.J. Dannenberg, S. Dapprich, A.D. Daniels, O. Farkas, J.B. Foresman, J.V. Ortiz, J. Cioslowski, D.J. Fox, Gaussian 09 Revision A.02, Gaussian Inc, Wallingford CT, 2009.

[24] T.H. Dunning Jr, Gaussian-basis sets for use in correlated molecular calculations. 1. The atoms boron through neon and hydrogen, J. Chem. Phys. 90 (1989) 1007-1023.

[25] (a) P.C. Hiberty, D. Danovich, A. Shurki, S. Shaik, Why does benzene possess a D6h symmetry? a theoretical approach based on the $\pi$-quasiclassical state for probing $\pi$-bonding energies, J. Am. Chem. Soc. 117 (1995) 7760-7768;

(b) K. Jug, P.C. Hiberty, S. Shaik, The $\sigma-\pi$ energy separation in modern electronic theory for ground states of conjugated systems, Chem. Rev. 101 (2001) 1477-1500.

[26] (a) S. Shaik, P. Maître, G. Sini, P.C. Hiberty, The charge-shift bonding concept. Electron pair bonds with very large resonance energies, J. Am. Chem. Soc. 114 (1992) 7861-7866;

(b) S. Shaik, Z. Chen, W. Wu, A. Stanger, D. Danovich, P.C. Hiberty, An excursion from normal to inverted $\mathrm{C}-\mathrm{C}$ bonds shows a clear demarcation between covalent and charge-shift bonds, ChemPhysChem 10 (2009) 2658-2669; (c) P.C. Hiberty, C. Megret, L. Song, W. Wu, S. Shaik, Barriers of hydrogen abstraction vs halogen exchange - an experimental manifestation of chargeshift bonding J. Am. Chem. Soc 128 (2006) 2836-2843;

(d) P.C. Hiberty, R. Ramozzi, L. Song, W. Wu, S. Shaik, The physical origin of large covalent-ionic energies in some two-electron bonds, Faraday Discuss. 135 (2007) 261-272;

(e) S. Shaik, D. Danovich, W. Wu, P.C. Hiberty, Charge-shift bonding and its manifestations in chemistry, Nat. Chem. 1 (2009) 443-449;

(f) B. Braida, P.C. Hiberty, The essential role of charge-shift bonding in hypervalent prototype XeF2, Nat. Chem. 5 (2013) 417-422.

[27] J.M. Galbraith, E. Blank, S. Shaik, P.C. Hiberty, П-bonding in second and third row molecules: testing the strength of Linus's blanket, Chem. Eur. J. 6 (2000) 2425-2434.

[28] (a) D.L. Cooper, J. Gerratt, M. Raimondi, Application of spin-coupled valence bond theory, Chem. Rev. 91 (1991) 929-964;

(b) D.L. Cooper, J.P. Gerratt, M. Raimondi, Modern valence bond theory, Adv. Chem. Phys. 69 (1987) 319-397;

(c) M. Sironi, M. Raimondi, R. Martinazzo, F.A. Gianturco, Recent developments of the SCVB method, in: D.L. Cooper (Ed.), Valence bond theory, Elsevier, Amsterdam, The Netherlands, 2002, pp. 261-277.

[29] E. Ploshnik, D. Danovich, P.C. Hiberty, S. Shaik, The nature of the idealized triple bonds between principal elements, and the $\sigma$-origins of trans-bent geometries - a valence bond study, J. Chem. Theory Comput. 7 (2011) 955968.

[30] (a) D. Danovich, P.C. Hiberty, W. Wu, H.S. Rzepa, The nature of the 4th bond in the ground state of $C_{2}$ : the quadruple bond conundrum, Chem. Eur. J. 20 (2014) 6220-6232;

(b) S. Shaik, D. Danovich, W. Wu, P. Su, H.S. Rzepa, P.C. Hiberty, Quadrupole bonding in C2 and analogous 8-valence electron species, Nat. Chem. 4 (2012) 195-200; 
(c) S. Shaik, H.S. Rzepa, R. Hoffmann, One molecule, two atoms, three views, four bonds?, Angew Chem. Int. Ed. 52 (2013) 3020-3033;

(d) G. Frenking, M. Hermann, Critical comments on "one molecule, two atoms, three views, four bonds?”, Angew. Chem. Int. Ed. 52 (2013) 5922 5925 ;

(e) D. Danovich, S. Shaik, R. Hoffmann, H.S. Rzepa, A response to the critical comments on "one molecule, two atoms, three views, four bonds?", Angew. Chem. Int. Ed. 52 (2013) 5926-5928;

(f) L.T. Xu, T.H. Dunning Jr., Insights into the perplexing nature of the bonding in C-2 from generalized valence bond calculations, J. Chem. Theory Comput. 10 (2014) 195-201.

[31] P. Su, J. Wu, W. Wu, S. Shaik, P.C. Hiberty, Bonding conundrums in the C2 molecule: a valence bond study, J. Chem. Theory Comput. 7 (2011) $121-130$
[32] One might be tempted to conclude that BOVB-calculated bonding energies might be larger than experimental values when using very large basis sets. It is however not so, if one retains the simplicity of the BOVB wave function, where each bond is described as one covalent and two ionic structures. Indeed, an important reason why large basis sets favor large bonding energies is that they allow angular electron correlation to be taken into account. For this to be possible in the BOVB framework, one would have to include additional VB structures. Thus, if one sticks to the simple form of BOVB wave functions, the bonding energy generally does not increase as the basis set is enlarged.

[33] Computational chemistry comparison and benchmark data base, NIST Standard Reference Data base 101, <http://www.cccbdb.nist.gov>.

[34] J. Toulouse, C.J. Umrigar, Full Optimization of Jastrow-Slater wavefunctions with application to the first-row atoms and homonuclear diatomic molecules, J. Chem. Phys. 128 (2008) 174101. 